\title{
Study on Identification Model of Cylinder Liner-Piston Ring Using Vibration Analysis Based on Fuzzy C-means Clustering
}

\author{
Zhiwei Guo ${ }^{*}, 1,2$, Chengqing Yuan ${ }^{1,2}$, Peng Liu ${ }^{1,2}$ \\ ${ }^{I}$ School of Energy and Power Engineering, Wuhan University of Technology, Wuhan, China \\ ${ }^{2}$ Key Laboratory of Marine Power Engineering and Technology, Ministry of Transportation, Wuhan, China
}

\begin{abstract}
Diesel engines have been widely used in various engineering applications. The performance of diesel engines is influenced greatly by the operation condition of the cylinder liner-piston ring. It is therefore crucial to investigate and assess the working condition of the cylinder liner-piston ring. Literature review indicated that there are few studies on this issue. Therefore, this study proposes a condition identification model for the cylinder liner-piston ring via fuzzy C-means clustering technique. The experiments of different surface treatment of cylinder and several typical fault modes were carried out using an internal combustion engine simulation tester. The fuselage vibration signals of the diesel engine were collected.The wavelet packet was then used to obtain the high frequency components of the vibration signal and extract its energy characteristics. The vibration identification model based on fuzzy C-means clustering has been established according to these characteristics. The analysis results show that the proposed model can identify the operating mode of cylinder liner-piston ring according to the vibration characteristics effectively.
\end{abstract}

Keywords: cylinder liner-piston ring, vibration analysis, characteristic information, identification model.

\section{INTRODUCTION}

Marine diesel engine is the heart of the vessel. The normal operation of marine diesel engines ensures the scheduled completion and efficiency of a trip. Any failures may result in significant economic losses and severe accidents. However, the engines often suffer from the deterioration of rubbing pairs $[1,2]$. The cylinder linerpiston ring is one of the most important rubbing pairs of the internal combustion engine, whose tribological condition has great influence on engine power, reliability, durability, economy and emissions. It is hence crucial to monitor condition of the typical tribological systems in a reliable and timely manner, which can detect undergoing faults in time to ensure the normal operation of the vessels. Additionally, it is very significant to extend the work reliability and life of cylinder liner-piston rings, improve the efficiency of diesel engine and fuel economy, and prolong service life through the study on the tribological problems of cylinder linerpiston ring [3].

Ryk and Kligerman [4] evaluated the effectiveness of micro-surface structure to improve tribological properties of reciprocating automotive components. $\mathrm{Fu}[5,6]$ showed that surface texturing with micro-pores could be used to maintain hydrodynamic effects even on nominally parallel surfaces. Optimal parameters for lubrication performance were also given. The area occupancy of micro-pores was $15 \%$, and the depth-diameter ratio was 0.5 . Lubrication oil thickness was

*Address correspondence to this author at the School of Energy and Power Engineering, Wuhan University of Technology, Wuhan 430063, P.R. China; Tel: +86-027-86549879; E-mail: guozhiwei.888@163.com increased by $10 \sim 15 \%$ and average friction force was decreased by $20 \%$ with bore processed cylinder. Burstein and Ingman [7] studied the lubrication model of the piston ring surface processing of random concave and regular concave. Rhode [8] concluded that the friction was reasonable if the length-diameter ratio was close to 0.14 .

Fuzzy clustering has been extensively used for performing tasks such as information retrieval [9], pattern recognition [10], and image processing [11]. Fuzzy clustering differs from hard clustering in the sense that it tries to split a data set into a number of overlapping subsets. The notion of fuzzyness can be thus found in the idea that data points are allowed to be part of multiple clusters.

There are many fuzzy clustering methods being introduced [12]. Fuzzy C-means clustering algorithm is one of the most important and popular fuzzy clustering algorithms. At present, the FCM algorithm has been extensively used in feature analysis, pattern recognition, image processing, classifier design [13, 14]. In this study, the fuzzy C-means clustering is adopted to identify the operation models of the diesel engine. A series of experiment tests have been done to show the efficacy of the fuzzy C-means clustering in the condition monitoring of diesel engines.

\section{EXPERIMENT AND METHODOLOGY}

\subsection{Experiments}

All experiments are conducted using a simulation tester [15], whose principle and image are illustrated in Fig. (1). The tester is designed for investigating the tribology and vibration characteristics of key parts in the internal combustion engine. A set of vibration sensors has been installed on tester fuselage to record the engine vibrations 

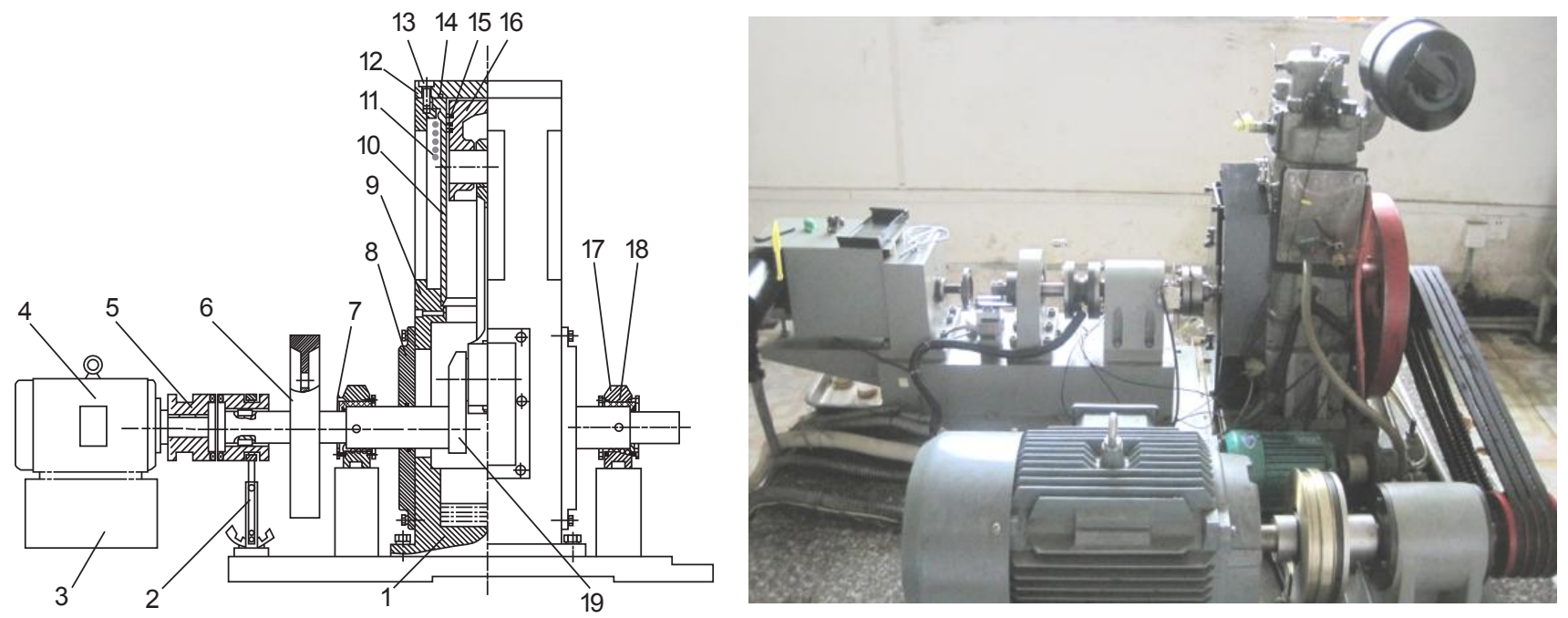

1-Oil pan 2-Clutch pedal 3-Cement base 4-Electromotor 5-Clutch 6-Flywheel 7-Left-side sliding bearing 8-End cover 9-Frame 10-Cyclinder 11-Resistance wire 12-Cylinder head 13-Bolt 14- 'O' shape sealing ring 15-Piston ring 16-Piston 17-Bearing seat 18-Right-side sliding bearing 19-Crankshaft

Fig. (1). The principle and image of the simulation tester.

from different locations and directions. A pipeline has been designed to collect the lubricant oil of the tester.
In the experiments, four kinds of cylinders, including the regular surface-concave cylinder, regular surface-groove cylinder, cylinder with concave and groove, and untreated cylinder, are prepared. The cylinders are composed of cast

Table 1. Geometric Models of Cylinders

\begin{tabular}{|c|c|c|c|}
\hline Distribution & & & 0 \\
\hline Parameters & $\begin{array}{l}\text { Concave dia. } 2 \mathrm{~mm} \\
\text { Concave depth } 200 \mu \mathrm{m}\end{array}$ & $\begin{array}{l}\text { Groove width } 1 \mathrm{~mm} \\
\text { Length } 20 \mathrm{~mm} \\
\text { Groove depth } 200 \mu \mathrm{m}\end{array}$ & $\begin{array}{c}\text { Concave dia. } 2 \mathrm{~mm} \\
\text { Concave depth } 200 \mu \mathrm{m} \\
\text { Groove width } 1 \mathrm{~mm} \text { Length } 20 \mathrm{~mm} \\
\text { Groove depth } 200 \mu \mathrm{m}\end{array}$ \\
\hline
\end{tabular}

Table 2. Images of Fault Modes

\begin{tabular}{|c|c|c|}
\hline Rocker Fracture & Bolt Fracture & Exfoliated Ejector Pin \\
\hline \hline & &
\end{tabular}


iron with the geometrical dimensions of $115 \mathrm{~mm}$ internal diameter and $105 \mathrm{~mm}$ stroke respectively. Geometric models of cylinders are shown in Table $\mathbf{1}$. The cylinder liner-piston ring has been tested using the mentioned cylinders, as well as several typical fault modes (such as the rocker fracture, bolt fracture and exfoliated ejector pin). Images of fault modes are illustrated in Table 2. The lubricant of the diesel engine is $15 \mathrm{~W} / 40 \mathrm{CD}$. The engine speeds set to $800 \mathrm{rpm}$ during the tests, vibration signal is collected every 20 minutes after running $2 h$ in each test conditions, the testing duration time was 8 hours and the sampling frequency is 20 $\mathrm{kHz}$.

\subsection{Signal Processing and Feature Extraction}

\subsubsection{The Wavelet Packet Decomposition}

During the measurement of actual mechanical signals, the mixture of noise signals increases the difficulty of fault feature extraction. The sources of noise are complicated. Possible reasons are mechanical defect or the occurrence of other vibration excitations. As a result, it is necessary to analyze and process the measured signals so that we can extract useful features [16].

The diesel engine is very complex system. The surface vibration of the diesel engine is caused by the interaction of its diverse internal excitations, including cylinder pressure, transverse impact of piston and reciprocating inertia force of crankshaft, etc. The cylinder vibration is caused by rocking of the piston. The impact of piston is high-frequency excitation. Therefore, it is worthy of analyzing the highfrequency signal of the diesel engine [17].

There are mainly three characteristic bands for vibration signal in power spectrum. The change in high frequency components is bigger than low frequency components. With the increase of test time, high frequency components increase.

The wavelet packet decomposition method [18] is adopted to analyze the high frequency of $2 \mathrm{kHz}-6 \mathrm{kHz}$. The original vibration signals are decomposed to four layers

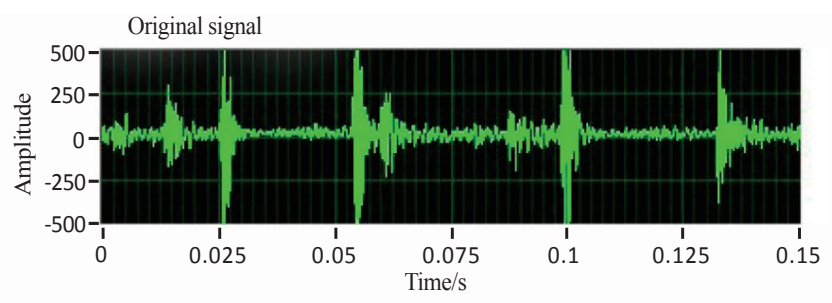

using wavelet packet decomposition. 16 wavelet sub-bands in the fourth layer are obtained. The fourth layer are arranged according to ascending order $X(4, i), i=0,1,2, \cdots 15$. It is required to reorder the coefficient after wavelet packet decomposition. In order to keep the main frequency consistent with $i$ monotonic increasing, the practical frequency bands reorder as $(4,0),(4,1),(4,3),(4,2),(4,6)$, $(4,7),(4,5),(4,4),(4,12),(4,13),(4,15),(4,14),(4$, $10),(4,11),(4,9),(4,8)$. Since the highest frequency of signal is $10 \mathrm{kHz}$, so the frequency of $2 \mathrm{kHz}$ is contained in wavelet packet node $(4,2)$. Because the high frequency components over $6 \mathrm{kHz}$ is so faint that it can be basically negligible, the high frequency vibration components of 2 $\mathrm{kHz}-6 \mathrm{kHz}$ are obtained through reconstructed the wavelet packet $(4,2)-(4,13)$. The vibration signals and power spectrum with regular surface-concave cylinder are show in Fig. (2), which include original signal and reconstructed signal after wavelet packet decomposition and reconstruction.

\subsubsection{Feature Extraction}

During the wavelet packet decomposition and reconstruction, useful characteristics of high frequency components on the vibration signal could be calculated. In the time domain, the root mean square (RMS) and kurtosis are extracted. The root mean square (RMS) and kurtosis are defined as follows:

Root mean square (RMS)

$$
\begin{gathered}
R M S=\sqrt{\frac{1}{n} \sum_{i=1}^{n} x_{i}^{2}} \\
\text { Kurtosis (KR) } \\
K R=\frac{n \sum_{i=1}^{n}\left(x_{i}-\bar{x}\right)^{4}}{\left(\sum_{i=1}^{n}\left(x_{i}-\bar{x}\right)^{2}\right)^{2}}
\end{gathered}
$$

where, $x_{i}(i=1, \ldots, \mathrm{n})$ is the signal value of sampling point

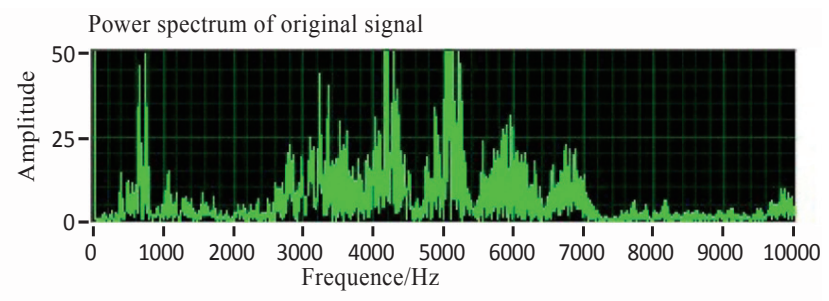

(a) Original signal
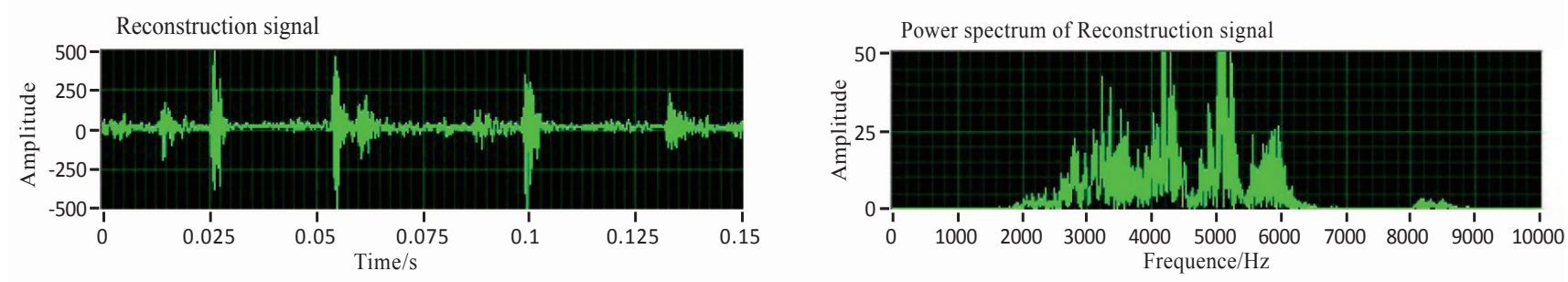

(b) Reconstructed signal

Fig. (2). The Vibration signals and power spectrum with regular surface-concave cylinder. 
on number $i, n$ is the number of sampling points, $\bar{x}$ is the average value.

Fourier transform method is the main analysis tool in frequency domain. It occupies an important position in the signal processing by building up a bridge between time domain and frequency domain. Feature vectors of frequency domain are extracted via Fourier transform. The extracted features are as follows:

Root mean square frequency (RMSF)

$R M S F=\sqrt{\frac{\sum_{k-1}^{K} f_{k}^{2} X_{k}}{\sum_{k=1}^{K} X_{k}}}$

Standard deviation frequency (STDF)

$S T D F=\sqrt{\frac{\sum_{k-1}^{K}\left(f_{k}-F C\right)^{2} X_{k}}{\sum_{k=1}^{K} X_{k}}}$

where, $X_{k}$ is the measurement of number $k$ in the spectrum signal, $K$ is the total number of spectral lines, $f_{k}$ is frequency value of number $k$ spectrum line, $F C$ is center frequency.

\section{FUZZY C- MEANS CLUSTERING}

\subsection{Fuzzy C-Means Clustering Algorithm}

FCM (Fuzzy c-means clustering) is known as fuzzy ISODATA. It is a clustering algorithm to determine the degree that each data point belongs to a cluster. Generally, traditional hard clustering algorithms use obvious boundaries to cluster different groups for the original data. To overcome this shortcoming, Bezdek offered the FCM algorithm improve the HCM (Hard c-means clustering) [19], where boundaries of different groups were allowed to be overlapped. The fuzzy logic was used to realize this.

FCM aims to classify vector $x_{i}(i=1,2, \ldots, n)$ into $c$ ambiguity group and calculate clustering center of each group. Fuzzy partition used in FCM is the mainly difference between fuzzy FCM and HCM. It determines the degree that each data point belongs to each group using membership value $0-1$. The element of membership matrix $U$ is allowed to be from 0 to 1 , which geared to Fuzzy partition. The total of membership in a data set is 1 based on the regulation of normalization.

$$
\sum_{i=1}^{c} u_{i j}=1, \forall j=1, \ldots, n
$$

where $\mathrm{c}$ is the number of ambiguity group.

Then, cost function (or objective function) of FCM is generalization form of equation (6):

$$
J\left(U, c_{1}, \ldots, c_{c}\right)=\sum_{i=1}^{c} J_{i}=\sum_{i=1}^{c} \sum_{j}^{n} u_{i j}^{m} d_{i j}^{2}
$$

where, $u_{\mathrm{ij}}$ is between 0 and $1, c_{i}$ is the clustering centers of fuzzy group $i, d_{\mathrm{ij}}=\left\|c_{\mathrm{i}}-x_{\mathrm{j}}\right\|$ is the Euclidean distance between the $i$ th clustering centers and the $j$ th data point, $x_{\mathrm{j}}$ is the $j$ th data point, $m \in[1, \infty]$ is weighted index.

New objective function is structured to obtain the necessary condition which makes equation (6) into minimum.

$$
\begin{aligned}
& \bar{J}\left(U, c_{1}, \ldots, c_{c}, \lambda_{1}, \ldots, \lambda_{n}\right) \\
& =J\left(U, c_{1}, \ldots, c_{c}\right)+\sum_{j=1}^{n} \lambda_{j}\left(\sum_{i=1}^{c} u_{i j}-1\right) \\
& =\sum_{i=1}^{c} \sum_{j}^{n} u_{i j}^{m} d_{i j}^{2}+\sum_{j=1}^{n} \lambda_{j}\left(\sum_{i=1}^{c} u_{i j}-1\right)
\end{aligned}
$$

where $\lambda_{j}, j=1,2, \ldots, n$ is Lagrange multiplier of $n$ constrained formula in equation (5). The necessary condition which makes equation (6) into minimum is then given by:

$$
c_{i}=\frac{\sum_{j=1}^{n} u_{i j}^{m} x_{j}}{\sum_{j=1}^{n} u_{i j}^{m}}
$$

and

$$
u_{i j}=\frac{1}{\sum_{k=1}^{c}\left(\frac{d_{i j}}{d_{k j}}\right)^{2 /(m-1)}}
$$

From the above two necessary condition, the FCM (Fuzzy c-mean) algorithm is a simply iterative process. To obtain the center $c_{i}$ and memberships matrix $U$ of each cluster by iteration, the following steps are adopted in FCM algorithm.

Step 1: initialize membership matrix $U$ using random number between 0 and 1 , and make it satisfy the constraint conditions equation (5); (8);

Step 2: calculate the clustering centers $c_{i}$ using equation

Step 3: calculate the cost function according to equation (6). The algorithm stops if it is less than a certain threshold value or it is less than a certain threshold value compared with the change of last cost function value;

Step 4: calculate next matrix $U$ based on equation (9), return to Step 2 .

From the above discussion, two parameters of cluster $c$ and weighted index $m$ are required in FCM algorithm. Ordinary $c$ is far less than total number of cluster sample, while it is ensured that $c>1 . m$ is a parameter which control the flexibility of algorithm, the effect of cluster will be poor if $m$ is too large while the algorithm will be close to HCM (Hard C mean clustering) if $m$ is too small.

\subsection{The Proposed Identification Model}

The fuzzy logic is very suitable for the condition identification of the diesel engines because that it is often difficult in determining the boundaries of different faults. The FCM is used to learn the relationship between the vibrations and the engine operation states. The FCM is trained by the input features of the engine vibration signals 


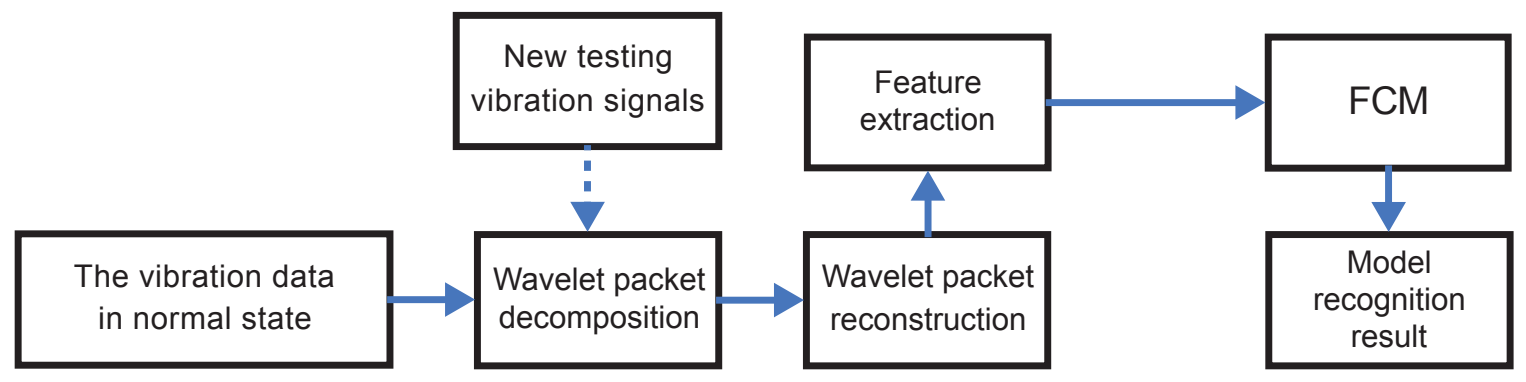

Fig. (3). The sketch diagram of the FCM based identification model.

to form an intelligent identification model. Then, the trained FCM identification model is tested by new input samples. The sketch diagram of the proposed identification model is illustrated as Fig. (3).

\section{VIBRATION IDENTIFICATION MODEL}

\subsection{The Establishment of Vibration Identification Model}

Characteristic parameters used in this study includes RMS, KR, RMSF, STDF, maximum amplitude and total vibration energy. Vibration identification model is established in which original cylinder, regular concave, regular groove, rocker fracture, bolt fracture and exfoliated ejector pin are taken as the output states of the cylinderpiston ring. Thirty samples have been prepared for each condition with the rotational speed of $800 \mathrm{rpm}$. A portion of the feature extraction results are shown in Table $\mathbf{3}$.

A training feature space $F R_{6 \times 180}$ is formed after the feature extraction. The column data are arranged in the order of RMS, KR, RMSF, STDF, maximum amplitude and total vibration energy. Cluster center of each state and memberships belong to each state are calculated using FCM (Fuzzy c-means clustering) algorithm. Vibration identification model is shown in Fig. (4).

Abscissa is the input data group in Fig. (4), each state has 30 samples, and it is arranged in the order of original cylinder liner, regular concave, regular groove, rocker fracture, bolt fracture and exfoliated ejector pin. The first group, 0 30 samples are characteristic values in the state of original cylinder liner. The second group, 31 60 samples are characteristic values in the state of regular concave. The third group, 61 90 samples are characteristic values in the state of regular groove. The forth group, 91 120 samples are characteristic values in the state of rocker fracture. The fifth group, 121 150 samples are characteristic values in the state of bolt fracture. The sixth group, 151 180 samples are characteristic values in the state of exfoliated ejector pin. Ordinate of Fig. (4) is the cluster center of each state. It can be seen that the training samples have been grouped into six clusters, and each sample has been identified correctly.

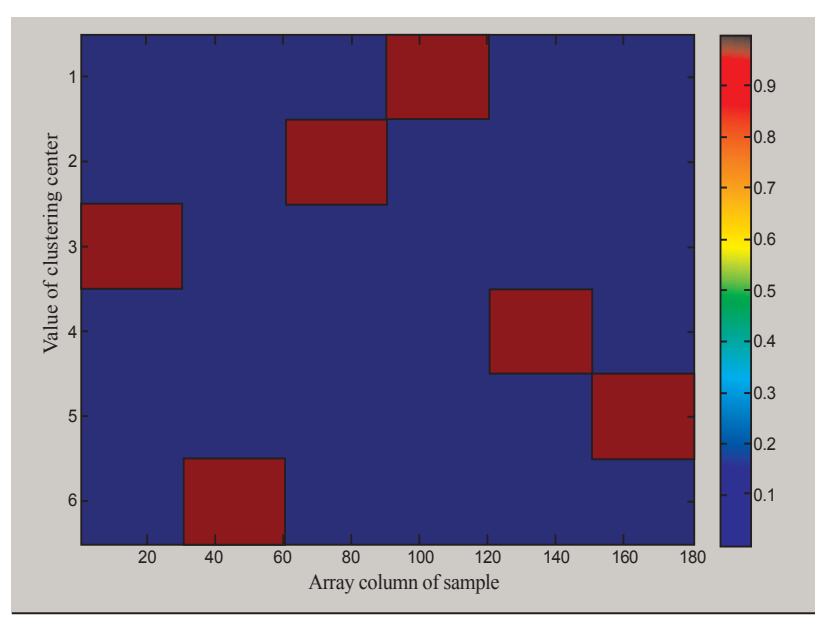

Fig. (4). Vibration identification model.

Taking this model as a benchmark, it only needs to add input feature vector into this model to determine the operation mode of other vibration signals. Then, the corresponding mode can be obtained by comparing clustering center values. Taking the vibration signals of regular concave as an example, the feature values is shown in Table 4. The corresponding relation after adding to this model is shown in Fig. (5).

It can be seen from Fig. (5) that the clustering center value of the testing data accords with the second group, so it belongs to the running state of regular concave. Thus, the identification result is correct.

Table 3. Eigenvalue of Vibration Signal

\begin{tabular}{|c|c|c|c|c|c|c|c|}
\hline $\mathbf{n} /\left(\mathbf{r} \cdot \mathbf{m i n}^{-1}\right)$ & type & RMS & KR & RMSF & STDF & Maximum Amplitude & Total Vibration Energy \\
\hline \hline \multirow{3}{*}{800} & Original cylinder & 1.0000 & 1.0000 & 1.0000 & 1.0000 & 1.0000 & 1.0489 \\
\cline { 2 - 8 } & Regular concave & 0.9301 & 0.6297 & 0.3820 & 1.0400 & 1.0000 \\
\cline { 2 - 8 } & Regular groove & 1.2062 & 1.4611 & 1.9296 & 1.0270 & 0.3867 \\
\cline { 2 - 8 } & Rocker fracture & 0.6001 & 0.8613 & 0.9125 & 0.9629 & 0.3566 & 3.7698 \\
\cline { 2 - 8 } & Bolt fracture & 0.9037 & 0.8663 & 1.1127 & 0.5671 & 0.3310 & 0.7742 \\
\cline { 2 - 8 } & Exfoliated ejector pin & 1.0064 & 0.5296 & 2.4959 & 1.0051 & 0.7649 & 1.3783 \\
\hline
\end{tabular}


Table 4. Vibration Signal of Regular Concave

\begin{tabular}{|c|c|c|c|c|c|c|}
\hline & RMS & KR & RMSF & STDF & Maximum Amplitude & Total Vibration Energy \\
\hline \hline Regular concave & 1.0728 & 0.6311 & 0.4211 & 1.1238 & 1.0531 & 0.4763 \\
\hline
\end{tabular}

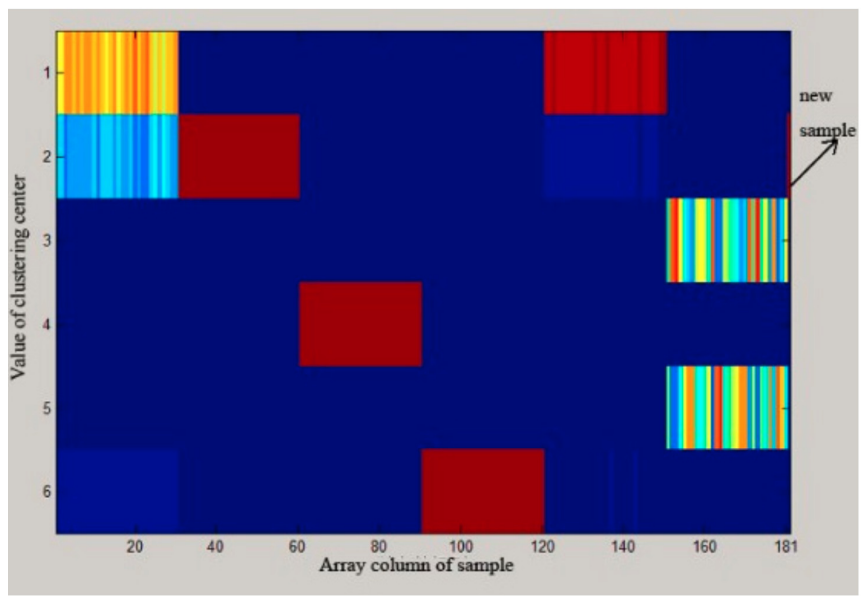

Fig. (5). Identification legend.

\subsection{Validation of Vibration Identification Model}

The running state of diesel engine can be obtained using this vibration identification model. Howerevr, the membership value is not equal to 1 , which is obtained only according to the maximum membership degree. It need to output the membership matrix and calculate the corresponding membership if the membership value is required.

Additional five samples for each condition are provided in Table 5, which belong to the running state of regular concave in $800 \mathrm{rpm}$. The membership matrix which is calculated based on this identification model is shown in Table 6. It properly identifies the correct state of cylinder liner-piston ring. As a result, the proposed FCM based identification model is efficient for the monitoring of diesel engines.

\section{SUMMARY}

The experiments are conducted using an internal combustion engine simulation tester for key rubbing pairs under different surface treatment of cylinder liner and several typical fault operation modes. The fuselage vibration signals during testing are collected and the wavelet packet decomposition techlonogy is used to obtain a high frequency signal components and extract its energy characteristics. Based on these characteristics, the vibration identification model is established using fuzzy C-means clustering. This model can identify the operating modes of cylinder liner-piston ring according to the characteristics of vibration signals.

\section{CONFLICT OF INTEREST}

The authors confirm that this article content has no conflict of interest.

\section{ACKNOWLEDGEMENTS}

The authors would like to express their sincere gratitude to the Natural Science Foundation of China (NSFC) for its funding the research projects (No: 51139005 and No: 50975213).

Table 5. Vibration Signal of Regular Concave

\begin{tabular}{|c|c|c|c|c|c|c|c|}
\hline $\mathbf{n} /\left(\mathbf{r} \cdot \mathbf{m i n}^{-1}\right)$ & & RMS & KR & RMSF & STDF & Maximum Amplitude & Total Vibration Energy \\
\hline \hline \multirow{3}{*}{800} & 1 & 1.0728 & 0.6311 & 0.4211 & 1.1238 & 1.0531 \\
\cline { 2 - 7 } & 2 & 0.9821 & 0.7374 & 0.4559 & 1.0738 & 1.0589 \\
\cline { 2 - 7 } & 3 & 0.9746 & 0.6971 & 0.5103 & 1.0556 & 1.1879 \\
\cline { 2 - 7 } & 4 & 0.9907 & 0.7286 & 0.4906 & 1.0414 & 1.0620 \\
\cline { 2 - 7 } & 5 & 0.9754 & 0.7426 & 0.4118 & 1.0488 & 0.5216 \\
\hline
\end{tabular}

Table 6. Membership Value of Vibration Identification

\begin{tabular}{|c|c|c|c|c|c|c|}
\hline \multirow{2}{*}{$\mathbf{n} /\left(\mathbf{r} \cdot \mathbf{m i n}^{-1}\right)$} & \multirow{2}{*}{ Type } & \multicolumn{5}{|c|}{ Class Number } \\
\hline & & 1 & 2 & 3 & 4 & 5 \\
\hline \multirow{6}{*}{$800 \mathrm{r} / \mathrm{min}$} & Original cylinder & 0.0058 & 0.0049 & 0.0028 & 0.0022 & 0.0065 \\
\hline & Regular concave & 0.9906 & 0.9919 & 0.9957 & 0.9964 & 0.9891 \\
\hline & Regular groove & $9.1 \times 10^{-5}$ & $7.5 \times 10^{-5}$ & $4.0 \times 10^{-5}$ & $3.1 \times 10^{-5}$ & 0.0001 \\
\hline & Rocker fracture & 0.0003 & 0.0003 & 0.0001 & 0.0001 & 0.0004 \\
\hline & Bolt fracture & $9.2 \times 10^{-5}$ & $7.6 \times 10^{-5}$ & $4.0 \times 10^{-5}$ & $3.1 \times 10^{-5}$ & 0.0001 \\
\hline & Exfoliated ejector pin & 0.0029 & 0.0026 & 0.0012 & 0.001 & 0.0037 \\
\hline
\end{tabular}




\section{REFERENCES}

[1] W. Yanxia and G. Hui, "Research on optimization for the piston pin and the piston pin boss", Open Mechanical Engineering Journal, vol. 5, pp.186-193, 2011

[2] F.K. Choy, V. Polyshchuk, J.J. Zakrajsek, R. F. Handschuh, D.P. Townsend, "Analysis of the effects of surface pitting and wear on the vibration of a gear transmission system", Tribology International, vol. 29, pp.77-83, 1996.

[3] K. Liu, Y.B. Xie and C.L. Gui, "A comprehensive study of the friction and dynamic motion of the piston assembly", Journal of Engineering Tribology, vol. 212, pp. 221-226, 1998.

[4] G. Ryk, Y. Kligerman and N.G. Buljuk. Experimental investigation of laser surface texturing for reciprocating automotive components. Tribology Transactions, vol. 45, pp. 444-449, 2002.

[5] Y.H. Fu and H.C. Lu, "Theoretical analysis on lubricaton and wear-resisting of the honed cylinder liner", Transactions of CSICE, vol. 24, pp. 559-564, 2006.

[6] Y.H. Fu and H.W. Zhang, "Numerical analysis on the lubrication performance of piston ring by surface micro-texturing", Transactions of CSICE, vol. 27, pp. 180-185, 2009.

[7] L. Burstein and D. Ingman, "Pore ensemble statistics in application to lubrication under reciprocating motion", Tribology Transactions, vol. 43, no. 2, pp. 205 - 212, 2000.

[8] S. M. Rhode. A mixed friction model for dynamically loaded contact with application for piston ring lubrication In: Proceedings of 7th Leeds-Lyon Symposium on Tribology, pp. 262-278, 1980.

[9] M.E.S. Mendes and L. Sacks. Assessment of the performance of fuzzy cluster analysis in the classification of RFC documents. In: Proceedings of LCS, London, UK 2000.

[10] D.E.A. Giles and R. Draeseke, "Econometric modeling using pattern recognition via the fuzzy C-means algorithm", In: Giles DEA, Ed. Computer-aided econometrics. Marcel Dekker: New York, 2001.
[11] H. Frigui and R. Krishnapuram, "A robust competitive clustering algorithm with applications in computer vision", IEEE Transactions on Pattern Analysis and Machine Intelligence, vol. 21, pp. 450-65. 1999.

[12] F. Hoppner, F. Klawonn, R. Kruse and T. Runkler. Fuzzy cluster analysis. Wiley Press, New York, 1999.

[13] M. C. Clark, L. O. Hall. MRI segmentation using fuzzy clustering techniques: integrating knowledge. Available at: http://www.csee. usf.edu/, 1995.

[14] Y. W. Lim and S. U. Lee, "On the color image segmentation algorithm based on the thresholding and the fuzzy c-means techniques" Pattern Recognition, vol. 23: pp. 935-951, 1990.

[15] X.P. Yan, C.Q. Yuan and Z.L. Liu, "Study of simulation tester for key rubbing pairs in internal-combustion engine", Advanced Materials Research, vol. 97, pp. 4359-4362, 2010.

[16] Z. Li, X. Yan, Z. Guo, P. Liu, C. Yuan and Z. Peng, "A new intelligent fusion method of multi-dimensional sensors and its application to tribo-system fault diagnosis of marine diesel engines" Tribology Letters, vol. 47, pp. 1-15, 2012.

[17] X.D. Dai, X.Y. Yuan and Y.B. Xie, "Coupling effect between dynamic behavior and lubricating behavior of cylinder-piston system in internal combustion engine" Tribology, vol. 23, no. 6, pp. 519-523, 2003.

[18] Z. Li, X. Yan, C. Yuan, Z. Peng and L. Li, "Virtual prototype and experimental research on gear multi-fault diagnosis using waveletautoregressive model and principal component analysis method", Mechanical Systems and Signal Processing, vol. 25, 2589-2607, 2011.

[19] Y.G. Hu, and L. Zhang, "Fault diagnosis and validity analysis for diesel engines with fuzzy c means clustering algorithm", Proceedings of the second international symposium on test automation \&instrumentation, vol. 3, pp. 1730-1734, 2008

(C) Guo et al.; Licensee Bentham Open.

This is an open access article licensed under the terms of the Creative Commons Attribution Non-Commercial License (http://creativecommons.org/licenses/by-nc/3.0/) which permits unrestricted, non-commercial use, distribution and reproduction in any medium, provided the work is properly cited. 